\title{
Clinical characteristics and genetic backgrounds of Japanese patients with atypical hemolytic uremic syndrome
}

\author{
Madoka Fujisawa ${ }^{1} \cdot$ Hideki Kato $^{1} \cdot$ Yoko Yoshida $^{1} \cdot$ Tomoko Usui $^{1} \cdot$ Munenori Takata $^{2} \cdot$ Mika Fujimoto $^{3}$. \\ Hideo Wada $^{4} \cdot$ Yumiko Uchida ${ }^{5} \cdot$ Koichi Kokame $^{5} \cdot$ Masanori Matsumoto $^{6} \cdot$ Yoshihiro Fujimura $^{7} \cdot$ Toshiyuki Miyata $^{8}$. \\ Masaomi Nangaku ${ }^{1}$
}

Received: 1 November 2017 / Accepted: 22 February 2018 / Published online: 6 March 2018

(c) The Author(s) 2019, corrected publication 2019

\begin{abstract}
Background Atypical hemolytic uremic syndrome (aHUS) is caused by complement overactivation, and its presentation and prognosis differ according to the underlying molecular defects. The aim of this study was to characterize the genetic backgrounds of aHUS patients in Japan and to elucidate the associations between their genetic backgrounds, clinical findings, and outcomes.

Methods We conducted a nationwide epidemiological survey of clinically diagnosed aHUS patients and examined 118 patients enrolled from 1998 to 2016 in Japan. We screened variants of seven genes related to complement and coagulation, as well as positivity for anti-CFH antibodies, and assessed clinical manifestations, laboratory findings, and clinical course. Results The most frequent genetic abnormalities were in $C 3(31 \%)$ and the frequency of $C F H$ variants was relatively low (10\%) compared to Western countries. The predominant variant in this cohort was C3 p.I1157T (23\%), which was related to favorable outcomes despite frequent relapses. A total of $72 \%$ of patients received plasma therapy, while $42 \%$ were treated with eculizumab. The prognosis of Japanese aHUS patients was relatively favorable, with a total mortality rate of $5.4 \%$ and a renal mortality rate of $15 \%$.

Conclusions The common occurrence of genotype $C 3$, especially the p.I1157T variant was the characteristic of the genetic backgrounds of Japanese aHUS patients that differed from those of Caucasian patients. In addition, the favorable prognosis of patients with the unique $C 3$ p.I1157T variant indicates that understanding the clinical characteristics of individual gene alterations is important for predicting prognosis and determining therapeutic strategies in aHUS.
\end{abstract}

Keywords Atypical hemolytic uremic syndrome $\cdot$ Thrombotic microangiopathy $\cdot$ Congenital disorder $\cdot$ Complement . Epidemiology

Electronic supplementary material The online version of this article (https://doi.org/10.1007/s10157-018-1549-3) contains supplementary material, which is available to authorized users.

Hideki Kato

hkatou-tky@umin.ac.jp

Masaomi Nangaku

mnangaku-tky@umin.ac.jp

1 Division of Nephrology and Endocrinology, The University of Tokyo, 7-3-1 Hongo, Bunkyo-ku, Tokyo 113-8655, Japan

2 Clinical Research Support Center (CresCent), The University of Tokyo, Tokyo, Japan

3 Department of Cardiology and Nephrology, Mie University Graduate School of Medicine, Mie, Japan
4 Department of Molecular and Laboratory Medicine, Mie University Graduate School of Medicine, Tsu, Mie, Japan

5 Department of Molecular Pathogenesis, National Cerebral and Cardiovascular Center, Suita, Japan

6 Department of Blood Transfusion Medicine, Nara Medical University, Nara, Japan

7 Japanese Red Cross Kinki Block Blood Center, Ibaraki, Japan

8 Department of Cerebrovascular Medicine, National Cerebral and Cardiovascular Center, Suita, Japan 


\section{Introduction}

Atypical hemolytic uremic syndrome (aHUS) is a severe systemic disease characterized by thrombocytopenia, hemolytic anemia, and acute renal injury, and is induced by complement overactivation in the alternative pathway [1]. Variants in the genes encoding complement factor $\mathrm{H}(\mathrm{CFH})$, complement factor I (CFI), complement $\mathrm{C} 3$, complement factor B (CFB), membrane cofactor protein (MCP), thrombomodulin (THBD), and diacylglycerol kinase $\varepsilon(D G K E)$ have been identified as inherited defects, and the production of antagonistic anti-CFH antibodies is a known acquired complement abnormality causing dysregulation of the complement pathway.

The pathogenesis of aHUS has been elucidated both by recent advances in genetic research and epidemiological studies in aHUS patients. The development of aHUS is considered to require the involvement of various environmental factors besides the underlying complement overactivation [2]. Furthermore, the clinical presentation and prognosis of aHUS differ depending on the underlying molecular abnormality. Therefore, genotype identification is clinically significant not only for aHUS diagnosis, but also for predicting each patient's treatment response and prognosis.

Racial and regional differences were suggested by our previous report, which showed a high incidence of $C 3$ variants in Japanese aHUS patients in a small cohort $[3,4]$. This finding suggests that aHUS patients in Japan have unique genetic backgrounds, though larger numbers of patients should be analyzed. Moreover, the influence of regional and racial differences on clinical characteristics is unclear and requires further study.

Against this background, this study aimed to document the genetic backgrounds and comprehensive characteristics of Japanese patients with initial onset aHUS, constituting the first large-scale cohort in East Asia. We clarified the racial differences in the genetic backgrounds of aHUS patients and the importance of understanding the clinical differences among individuals with various underlying abnormalities.

\section{Materials and methods}

\section{Patients}

We enrolled Japanese patients clinically diagnosed with aHUS from 1998 to 2016 as part of a research project with the support of the Ministry of Health, Labour, and Welfare of Japan. Diagnoses were based on Japanese guidelines for
aHUS [5] (see Online Resources). Clinical and laboratory data were retrospectively collected from consultation letters or questionnaires. ESRD was defined as severe renal impairment requiring renal replacement therapy (RRT). Remission was defined as hematological remission (platelet counts above $10 \times 10^{4} / \mu 1$ and lactate dehydrogenase (LDH) levels below $222 \mathrm{U} / \mathrm{l}$ ) with preserved renal function (not reaching ESRD).

\section{Genetic analysis and variant interpretation}

Genetic analysis was performed at the Department of Molecular Pathogenesis of the National Cerebral and Cardiovascular Center, as previously described [6, 7] (see Online Resources). Variants with amino acid substitutions in exon sequences, whose minor allele frequency (MAF) scores were $<0.005$ in international databases (see Online Resources), were designated as candidate aHUS-predisposing variants.

\section{Autoantibodies against CFH}

The presence of anti-CFH autoantibodies in each patient's plasma or serum was evaluated using CFH IgG ELISA kits (Abnova, Taipei, Taiwan) according to the manufacturer's instructions. Positivity was also confirmed by Western blot analysis, as previously described [6].

\section{Statistical analysis}

Statistical analysis was performed with JMP® Pro 11.2 (SAS Institute Inc., Cary, NC, USA). Results are expressed as numerical values and median (range) for continuous variables and as percentages for categorical variables. Differences in clinical presentations and laboratory data among patients with $C 3$ p.I1157T and other $C 3$ variants were analyzed by chi-squared or Fisher tests. Cumulative renal survival rates were estimated by Kaplan-Meier analyses, excluding dead patients, and the log-rank test was used to compare these rates between $C 3$ p.I1157T and other $C 3$ variants. Differences were considered statistically significant at $p<0.05$.

\section{Ethics statement}

This study was approved by the ethical committee of The University of Tokyo Hospital (IRB G10029) and registered to UMIN-CTR (UMIN000014869). It was performed in accordance with the 1964 Declaration of Helsinki and its later amendments, and the Ethical Guidelines on Clinical Studies of the Ministry of Health, Labour and Welfare of Japan. Written informed consent was obtained from all patients or from their parents. 


\section{Results}

\section{Demographic backgrounds}

One hundred eighteen Japanese patients from 103 families were clinically diagnosed with aHUS from 1998 to 2016 (Table 1). Initial onset occurred at a median age of 6 years, ranging from 3 months to 84 years, and during childhood $(<18$ years of age) in $65 \%$ of cases. Seventy-six patients were males (64\%), and this male predominance existed regardless of age (52 males and 25 females in childhood, and 24 males and 17 females in adulthood).

\section{Clinical manifestations at the initial onset of aHUS}

Clinical manifestations of each patient were evaluated at their first visit for aHUS symptoms (Table 1, left column). Overall, $75 \%$ of patients experienced possible triggering events before hospitalization. Among patients whose laboratory data were available, $25 \%$ did not manifest all three triads of thrombotic microangiopathy. Anemia $(\mathrm{Hb}$ levels $<10 \mathrm{~g} / \mathrm{dl}$ ) was observed in $75 \%$ of patients and severe anemia $(<6 \mathrm{~g} / \mathrm{dl})$ was seen in $18 \%$. Increased levels of LDH ( $>222 \mathrm{U} / \mathrm{l})$ were found in all patients, but they ranged widely, from 276 to $8800 \mathrm{U} / \mathrm{l}$. Thrombocytopenia (platelet count $<15 \times 10^{4} / \mu \mathrm{l}$ ) occurred in $97 \%$ of patients, and was severe $\left(<3 \times 10^{4} / \mathrm{l}\right)$ in as many as $42 \%$. All patients demonstrate acute kidney injury (except three already diagnosed with ESRD), and $48 \%$ required RRT during hospitalization. Decreased levels of C3 $(<73 \mathrm{mg} /$ dl) were found in $47 \%$ of patients. Most patients demonstrated severe proteinuria and hematuria. The median proteinuria level was $11.7 \mathrm{~g} / \mathrm{g}$ Cre, and $44 \%$ of patients developed nephrotic syndrome. Moreover, $98 \%$ of patients showed dipstick-positive hematuria, which was confirmed in $85 \%$ of cases by microscopic evaluation.

The coagulation-fibrinolysis system was evaluated based on the Japanese criteria for diagnosis of disseminated intravascular coagulopathy (DIC) [8] (Fig. 1). We found that $72 \%$ of patients showed increased levels of fibrinogen/fibrin degradation products (FDP) (>10 mg/ dl). However, at their initial presentation, only $13 \%$ of patients had a mildly elevated international normalized ratio of prothrombin time (PT-INR) $(>1.25)$, 8\% demonstrated mildly decreased fibrinogen levels (from 100 to $150 \mathrm{mg} / \mathrm{dl}$ ), and $2 \%$ exhibited severely decreased fibrinogen $(<100 \mathrm{mg} / \mathrm{dl})$.

\section{Genetic and acquired abnormalities in patients with aHUS in Japan}

We screened seven genes and anti-CFH autoantibodies, all related to the development of aHUS, in 104 patients, whose samples were available. Twenty-seven variants were detected in 48 patients (46\%) (Table 2). Anti-CFH autoantibodies were identified in 20 patients, none of whom demonstrated pathogenic or rare gene variants in the seven analyzed genes. Patients were classified into the following six groups according to the identified abnormalities: $\mathrm{C} 3$, 32 of 104 patients (31\%); CFH, 10 (10\%); MCP, $5(5 \%)$; DGKE, 1 (1\%); anti-CFH autoantibody, 20 (19\%); and unidentified, 36 (35\%). No rare, non-synonymous variants in $C F B$ or $C F I$ were detected.

Genetic testing revealed that compared to Western countries, the frequency of $C F H$ variants in Japan (10\%) was lower [2,9] while that of $C 3$ variants was higher $(31 \%)$. Of note, the $C 3$ p.I1157T variant was detected in 24 patients (23\%) from 16 families.

Six patients had combined mutations: three carrying $C 3$ p.I1157T also had heterozygous $M C P$ variants with unknown pathogenicity; two from the same family had $M C P$ p.T98I, and one had MCP p.P195S. One patient had THBD p.T500M in addition to $C F H$ p.R1215Q, the latter of which is located in a mutational hotspot region of $\mathrm{CFH}$ and was reported to impair function [10]. One patient had a combination of two heterozygous $M C P$ variants, p.Y189D and p.A359V, the first of which was shown to be predisposing [11]. We screened for $D G K E$ variants in 22 patients under 2 years $[12,13]$ and identified one patient $(5 \%)$ with compound heterozygous variants $[7,14]$.

\section{Clinical characteristics according to complement abnormalities}

We then compared the clinical characteristics of aHUS patients according to genetic or acquired backgrounds. Age at the initial aHUS onset differed depending on the underlying abnormality, but the initial episode usually occurred during childhood in the $\mathrm{C} 3, \mathrm{MCP}$, and unidentified groups (Table 1 and Online Resource 1). Patients with anti-CFH antibodies showed a biphasic distribution of age at aHUS onset: during childhood (from ages 4 to 11 ) in $80 \%$ and during middle-to-old age in the remaining $20 \%$. Males were more commonly affected than females in all groups. A family history of aHUS was frequent in the $\mathrm{C} 3(47 \%)$ and $\mathrm{MCP}$ (40\%) groups, was rare in the $\mathrm{CFH}(10 \%)$ and the unidentified (3\%) groups, and was absent in the DGKE and anti-CFH antibody groups. Although most laboratory data at the first visit after initial aHUS onset did not differ between groups, severe anemia was observed in patients with $C F H$ variants (38\%), and the ratio of patients with reduced C3 levels 
Table 1 Demographics of aHUS patients in Japan and clinical and biological characteristics at first onset: stratification by complement abnormalities

\begin{tabular}{|c|c|c|c|c|c|c|c|c|}
\hline \multirow[t]{2}{*}{ Characteristics } & \multirow[t]{2}{*}{ Overall } & \multicolumn{4}{|c|}{ Genetic abnormalities } & \multirow[t]{2}{*}{ Anti-CFH Abs } & \multirow[t]{2}{*}{ Unidentified } & \multirow[t]{2}{*}{ Unanalyzed } \\
\hline & & $C 3$ & $\mathrm{CFH}$ & $M C P$ & $D G K E$ & & & \\
\hline Patients $(n)$ & 118 & 32 & 10 & 5 & 1 & 20 & 36 & 14 \\
\hline $\begin{array}{l}\text { Age at initial } \\
\text { onset (years) }\end{array}$ & $6.0(0.3-84.0)$ & $6.0(0.3-70.0)$ & $\begin{array}{l}26.5(0.3- \\
75.0)\end{array}$ & $6.0(1.0-50.0)$ & 0.3 & $6.0(4.0-75.0)$ & $\begin{array}{l}15.0(0.3- \\
80.0)\end{array}$ & $4.0(0.5-84.0)$ \\
\hline $\begin{array}{l}\text { Children } \\
\quad(<18 \text { years of } \\
\text { age })(\%)\end{array}$ & 65 & 66 & 20 & 80 & 100 & 80 & 56 & 93 \\
\hline Male (\%) & 64 & 66 & 60 & 100 & 100 & 60 & 64 & 57 \\
\hline $\begin{array}{l}\text { Family history of } \\
\text { HUS }(\%)\end{array}$ & 25 & 47 & 10 & 40 & 0 & 0 & 3 & 79 \\
\hline \multicolumn{9}{|c|}{ Clinical characteristics } \\
\hline $\begin{array}{l}\text { Probable trigger } \\
\text { events }(\%)\end{array}$ & 75 & 83 & 67 & 100 & 100 & 72 & 74 & 75 \\
\hline $\begin{array}{l}\text { Gastrointesti- } \\
\text { nal infections }\end{array}$ & 21 & 17 & 33 & 0 & 0 & 28 & 21 & 0 \\
\hline $\begin{array}{r}\text { Respiratory } \\
\text { infections }\end{array}$ & 20 & 17 & 0 & 0 & 0 & 39 & 18 & 25 \\
\hline Influenza & 5 & 0 & 11 & 100 & 0 & 0 & 0 & 25 \\
\hline $\begin{array}{l}\text { Other infec- } \\
\text { tions }\end{array}$ & 13 & 25 & 11 & 0 & 100 & 6 & 9 & 25 \\
\hline Others & 16 & 25 & 11 & 0 & 0 & 0 & 26 & 0 \\
\hline $\begin{array}{l}\text { Duration } \\
\text { between } \\
\text { onset and } \\
\text { first visit } \\
\text { (days) }\end{array}$ & $5(0-45)$ & $3(0-8)$ & $4(0-30)$ & 3 & 3 & $6(1-30)$ & $7(0-45)$ & $1(0-2)$ \\
\hline \multicolumn{9}{|c|}{ Physiological manifestations (\%) } \\
\hline Diarrhea & 23 & 17 & 33 & 50 & 0 & 25 & 24 & 0 \\
\hline Bloody stool & 6 & 0 & 11 & 0 & 0 & 0 & 12 & 0 \\
\hline $\begin{array}{l}\text { Nausea or vom- } \\
\text { iting }\end{array}$ & 47 & 17 & 67 & 50 & 100 & 60 & 44 & 40 \\
\hline Fever & 52 & 42 & 44 & 100 & 100 & 40 & 59 & 60 \\
\hline $\begin{array}{l}\text { Central nervous } \\
\text { system mani- } \\
\text { festations }\end{array}$ & 24 & 8 & 33 & 100 & 0 & 32 & 21 & 20 \\
\hline Purpura & 24 & 8 & 11 & 50 & 0 & 40 & 21 & 40 \\
\hline Macrohematuria & 18 & 42 & 0 & 50 & 0 & 25 & 3 & 60 \\
\hline Oligo-anuria & 37 & 33 & 44 & 0 & 0 & 10 & 56 & 40 \\
\hline \multicolumn{9}{|c|}{ Serological evaluation } \\
\hline $\mathrm{Hb}(\mathrm{g} / \mathrm{dl})$ & $7.4(3.2-14.5)$ & $8.1(5.3-12.7)$ & $7.2(5.0-14.5)$ & $\begin{array}{l}10.2(10.0- \\
10.3)\end{array}$ & 8.9 & $6.4(4.2-13.5)$ & $7.6(3.2-13.2)$ & $9.1(7.0-11.0)$ \\
\hline $\begin{array}{l}\mathrm{Hb}<10 \mathrm{~g} / \mathrm{dl} \\
(\%)\end{array}$ & 75 & 62 & 75 & 0 & 100 & 89 & 75 & 75 \\
\hline $\begin{array}{c}\mathrm{Hb}<6 \mathrm{~g} / \mathrm{dl} \\
(\%)\end{array}$ & 18 & 8 & 38 & 0 & 0 & 16 & 22 & 0 \\
\hline Plt $\left(\times 10^{4} / \mu \mathrm{l}\right)$ & $3.4(0.2-24.4)$ & $3.9(1.3-9.8)$ & $3.0(1.2-7.4)$ & $4.7(2.0-7.3)$ & 4.1 & $2.6(0.2-11.5)$ & $4.0(0.8-24.4)$ & $4.1(1.1-8.5)$ \\
\hline $\begin{array}{l}\mathrm{Plt}<15 \times 10^{4} / \\
\quad \mu \mathrm{l}(\%)\end{array}$ & 97 & 100 & 100 & 100 & 100 & 100 & 94 & 100 \\
\hline $\begin{array}{c}\text { Plt }<3 \times 10^{4} / \\
\mu l(\%)\end{array}$ & 42 & 46 & 50 & 50 & 0 & 58 & 28 & 50 \\
\hline $\mathrm{LDH}(\mathrm{U} / \mathrm{l})$ & $\begin{array}{l}2051(276- \\
8800)\end{array}$ & $\begin{array}{l}3069(1165- \\
6290)\end{array}$ & $\begin{array}{l}2009(1261- \\
3940)\end{array}$ & $\begin{array}{l}2156(428- \\
3884)\end{array}$ & 1830 & $\begin{array}{l}2983(299- \\
8800)\end{array}$ & $\begin{array}{l}1470(392- \\
4511)\end{array}$ & $\begin{array}{l}2323(276- \\
7966)\end{array}$ \\
\hline
\end{tabular}


Table 1 (continued)

\begin{tabular}{|c|c|c|c|c|c|c|c|c|}
\hline \multirow[t]{2}{*}{ Characteristics } & \multirow[t]{2}{*}{ Overall } & \multicolumn{4}{|c|}{ Genetic abnormalities } & \multirow[t]{2}{*}{ Anti-CFH Abs } & \multirow[t]{2}{*}{ Unidentified } & \multirow[t]{2}{*}{ Unanalyzed } \\
\hline & & C3 & CFH & $M C P$ & $D G K E$ & & & \\
\hline $\begin{array}{l}\text { Increased } \\
\text { LDH (\%) }\end{array}$ & 100 & 100 & 100 & 100 & 100 & 100 & 100 & 100 \\
\hline \multicolumn{9}{|l|}{ Renal function } \\
\hline $\begin{array}{c}\text { eGFR }(\mathrm{ml} / \mathrm{min} \\
\left.\text { per } 1.73 \mathrm{~m}^{2}\right)\end{array}$ & $\begin{array}{c}19.5(2.2- \\
105.3)\end{array}$ & $\begin{array}{l}13.0(7.5- \\
62.6)\end{array}$ & $\begin{array}{l}14.5(2.7- \\
25.1)\end{array}$ & $\begin{array}{l}24.0(2.2- \\
45.8)\end{array}$ & 13.4 & $\begin{array}{l}31.5(8.6- \\
56.9)\end{array}$ & $\begin{array}{c}16.8(4.0- \\
105.3)\end{array}$ & $\begin{array}{l}50.7(21.1- \\
69.8)\end{array}$ \\
\hline BUN (mg/dl) & $\begin{array}{l}60.0(11.7- \\
174.0)\end{array}$ & $\begin{array}{l}54.0(24.3- \\
134.0)\end{array}$ & $\begin{array}{l}56.0(31.2- \\
121.0)\end{array}$ & $\begin{array}{l}71.9(41.0- \\
102.7)\end{array}$ & 38.8 & $\begin{array}{l}64.8(28.0- \\
136.7)\end{array}$ & $\begin{array}{c}60.5(11.7- \\
174.0)\end{array}$ & $\begin{array}{l}38.5(30.0- \\
54.4)\end{array}$ \\
\hline AKI (\%) & 99 & 100 & 100 & 100 & 100 & 100 & 97 & 100 \\
\hline $\begin{array}{l}\text { AKI requiring } \\
\text { RRT }(\%)\end{array}$ & 48 & 44 & 70 & 67 & 100 & 26 & 55 & 50 \\
\hline \multicolumn{9}{|c|}{ Complement evaluation } \\
\hline C3 (mg/dl) & $74(14-145)$ & $68(42-99)$ & $87(35-114)$ & $79(46-111)$ & 20 & $48(14-121)$ & $93(28-145)$ & $56(16-95)$ \\
\hline $\begin{array}{l}\text { Reduced C3 } \\
(\%)\end{array}$ & 47 & 56 & 25 & 50 & 100 & 89 & 23 & 50 \\
\hline $\mathrm{C} 4(\mathrm{mg} / \mathrm{dl})$ & $23(8-45)$ & $28(10-38)$ & $29(8-45)$ & $23(17-28)$ & 14 & $25(9-37)$ & $22(10-44)$ & $31(19-44)$ \\
\hline $\begin{array}{l}\text { Reduced C4 } \\
(\%)\end{array}$ & 8 & 14 & 13 & 0 & 0 & 6 & 7 & 0 \\
\hline \multicolumn{9}{|l|}{ Urinary test } \\
\hline $\begin{array}{l}\text { Positive occult } \\
\text { blood (\%) }\end{array}$ & 98 & 100 & 100 & NA & NA & 100 & 95 & 100 \\
\hline Hematuria (\%) & 85 & 100 & 100 & NA & NA & 86 & 78 & NA \\
\hline $\begin{array}{l}\text { Positive pro- } \\
\text { teinuria (\%) }\end{array}$ & 98 & 100 & 100 & NA & NA & 100 & 96 & 100 \\
\hline $\begin{array}{l}\text { Urinary protein } \\
\text { (g/gCre) }\end{array}$ & $\begin{array}{l}11.7(0.6- \\
36.3)\end{array}$ & $\begin{array}{l}11.6(8.2- \\
14.5)\end{array}$ & $\begin{array}{l}13.7(3.9- \\
35.9)\end{array}$ & NA & NA & $\begin{array}{l}11.5(4.1- \\
36.3)\end{array}$ & $\begin{array}{l}13.0(0.6- \\
28.5)\end{array}$ & NA \\
\hline $\begin{array}{l}\text { Nephrotic syn- } \\
\text { drome }(\%)\end{array}$ & 44 & 50 & 60 & NA & NA & 13 & 55 & 0 \\
\hline
\end{tabular}

Results are expressed as numerical values, medians (range) for continuous variables, and percentages for categorical variables unless otherwise indicated. Increased LDH is defined as LDH $>222 \mathrm{U} / \mathrm{l}$. Reduced C3 and C4 are defined as $<73$ and $<11 \mathrm{mg} / \mathrm{dl}$, respectively

HUS hemolytic uremic syndrome, $A b s$ antibodies, $H b$ hemoglobin, $P l t$ platelets, $L D H$ lactate dehydrogenase, $e G F R$ estimated glomerular filtration rate, $B U N$ blood urea nitrogen, $A K I$ acute kidney injury, $R R T$ renal replacement therapy, Cre creatinine, NA not available

varied by abnormality. Approximately half of the patients with $C 3, M C P$ variants, one patient with $D G K E$ variant, and $89 \%$ of the patients with anti-CFH antibody showed decreased levels of $\mathrm{C} 3$.

\section{Treatment at the initial onset of aHUS and subsequent outcomes}

Information on treatment administered at the initial onset of aHUS was available in 101 patients (Table 3). Seventy-four patients were treated with plasma therapy at a median of 2 days (1-38 days) after admission, including 33 who began taking eculizumab at a median of 10 days (1-181 days) after starting plasma therapy. One patient was treated with eculizumab only. Fifty-two patients were diagnosed with aHUS after eculizumab was approved for the treatment of aHUS in Japan in September 2013. Thereafter, the overall treatment approach to aHUS changed and 54\% (28/52) of patients were treated with eculizumab.

The mortality rate of aHUS patients in the acute phase was $3.3 \%$ and did not differ according to abnormality. The overall renal mortality rate at discharge was $12.8 \%$, but was higher in $\mathrm{CFH}$ variants $(38 \%)$ and the unidentified group (24\%). The risk of death or ESRD in the acute phase did not differ by treatment choice for any abnormality (Online Resource 2).

\section{Long-term outcomes}

Patients were followed for a median of 2.5 years (1 month to 54 years) after the initial onset of aHUS, both retrospectively and prospectively in 104 patients. The total mortality rate was $5.4 \%$, with two patients dying within a year after hospital discharge (Table 3 and Online Resource 3), and the renal mortality rate was $15 \%$ (Fig. 2 
Fig. 1 Distribution of parameters regarding coagulation and fibrinolytic factors of aHUS patients at the initial visit. Each level is expressed as median (range). a PT-INR $(n=60)$ : 1.07 (0.76-1.44); eight patients with PT-INR $>1.25$. b APTT $(n=59): 29.5(15.3-67.1)$. c Fibrinogen $(n=53)$ : 282 (76-572); five patients with fibrinogen $<150 \mathrm{mg} / \mathrm{dl}$. d FDP $(n=53)$ : $14.6(0.5-194.0) ; 15$ patients with FDP $>10 \mu \mathrm{g} / \mathrm{dl}$. $P T-I N R$ international normalized ratio of prothrombin time, $A P T T$ activated partial thromboplastin time, FDP fibrinogen/ fibrin degradation products
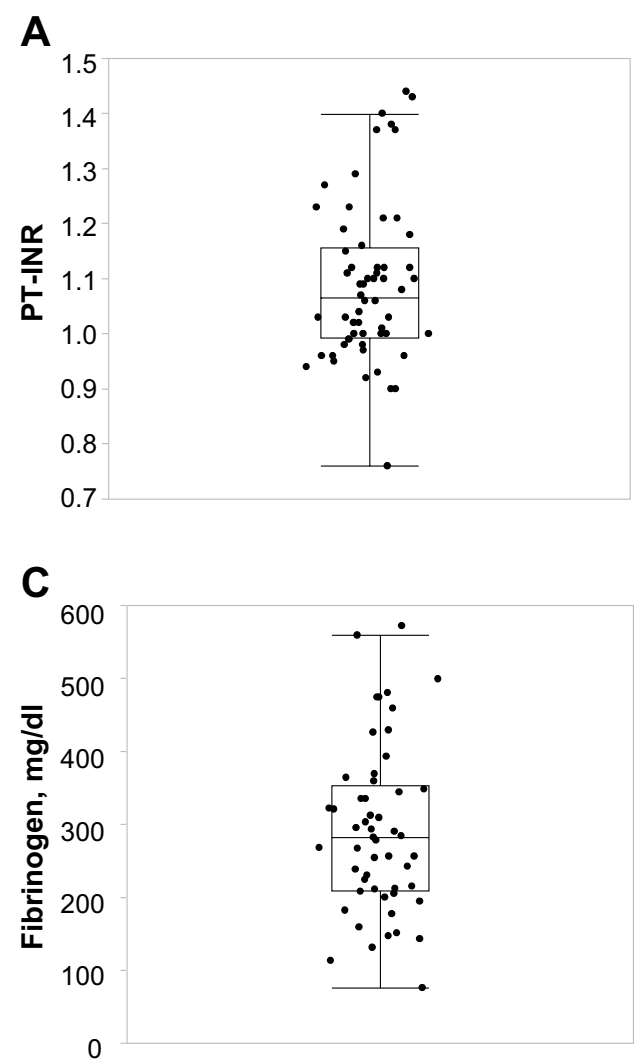
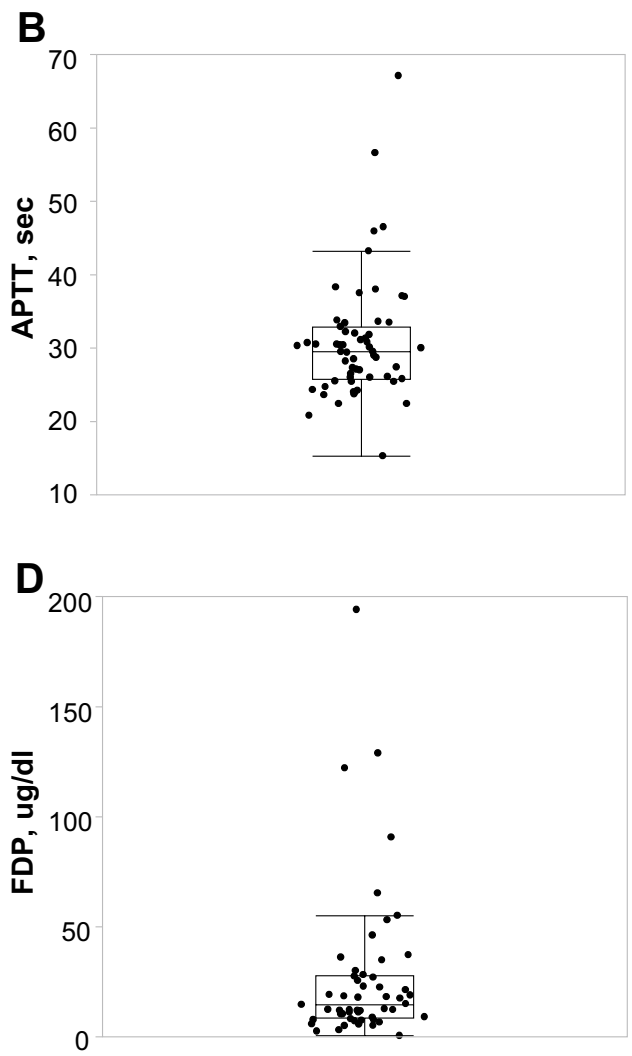

and Online Resource 4). Relapses occurred in patients with DGKE (100\%), C3 (77\%), MCP (50\%), and $C F H$ (38\%) variants.

Forty-one patients received eculizumab therapy, including those treated for relapses. Eculizumab was discontinued in 13 patients, 12 of whom maintained remission for a median of 1 year (0.5-3 years), while one patient with $C 3$ p.I1157T relapsed and resumed eculizumab treatment [15] (Online Resource 5). All 27 patients who received eculizumab as maintenance therapy were free from relapses, ESRD, and death, except for the single patient with a $D G K E$ variant.

Among the 20 patients with anti-CFH antibodies, 8 were treated with immunosuppressive medication, 5 with observational therapy, and 4 with eculizumab, each as maintenance therapy. Two patients were able to withdraw from RRT (Online Resource 4) more than a year after aHUS onset, and ultimately all 20 patients were free from relapse, ESRD, and death.

Renal transplantation was performed in three patients, all of whom experienced aHUS relapse after transplantation. One with $C 3$ variant redeveloped ESRD, while the other two, one with CFH variant and the other in the unidentified group, recovered renal function (Online Resource 4).

\section{C3 p.I1157T variant compared to other C3 variants}

The clinical characteristics of patients with the $C 3$ p.I1157T variant differed in several ways from those of patients with other $C 3$ variants (Table 4). Interestingly, reduced C3 levels at aHUS onset were observed in none of the patients with $C 3$ p.I1157T but all of those with other $C 3$ variants (Table 4). The median number of relapse episodes in patients with $C 3$ p.II157T was significantly higher than in those with other $C 3$ variants (2 vs. $0, p=0.016$ ). However, patients carrying $C 3$ p.II157T showed better outcomes, both in the acute phase ( $p=0.041$, Table 4$)$ and during long-time followup ( $p=0.023)$. Furthermore, many $C 3$ p.I1157T patients achieved remission with only supportive care $(65 \%)$ or plasma therapy $(35 \%)$.

\section{Discussion}

We report here a nationwide aHUS cohort in Japan in the first study from East Asia to include a substantial number of patients, as well as one of the largest global aHUS cohort series. We included 118 patients clinically diagnosed with aHUS over the last two decades and confirmed higher frequencies of $C 3$ variants and anti-CFH antibodies and lower 
Table 2 Summary of 27 variants identified in patients with aHUS

\begin{tabular}{|c|c|c|c|c|c|c|c|}
\hline \multirow[t]{2}{*}{ Gene } & \multirow[t]{2}{*}{ Patient code } & \multirow[t]{2}{*}{ Inheritance } & \multirow[t]{2}{*}{ SNP rs } & \multirow[t]{2}{*}{ Nucleotide } & \multirow[t]{2}{*}{ Predicted consequence } & \multicolumn{2}{|c|}{ MAF score } \\
\hline & & & & & & HGVD & $\begin{array}{l}1000 \\
\text { Genomes } \\
\text { phase } 3\end{array}$ \\
\hline \multirow[t]{9}{*}{ C3 } & TC32 & Heterozygous & - & c.535T $>C$ & p.S179P & - & - \\
\hline & ТC28 & Heterozygous & - & c. $544 \mathrm{~T}>\mathrm{C}$ & p.S182P & - & - \\
\hline & TC4 & Heterozygous & - & c. $640 \mathrm{C}>\mathrm{T}$ & p.P214S & - & - \\
\hline & $2 \mathrm{H}$ & Heterozygous & rs200967589 & c. $1273 \mathrm{C}>\mathrm{T}$ & p.R425C & 0.0022 & 0.0006 \\
\hline & TC3 & Heterozygous & rs767334972 & c. $1663 \mathrm{G}>\mathrm{A}$ & p.V555I & - & - \\
\hline & TC42 & Heterozygous & - & c. $3125 \mathrm{G}>\mathrm{T}$ & p.R1042L & - & - \\
\hline & 30 & Heterozygous & - & c. $3313 \mathrm{~A}>\mathrm{C}$ & p.K1105Q & - & - \\
\hline & $\begin{array}{c}\mathrm{F} 1, \mathrm{~F} 2, \mathrm{~F} 3, \mathrm{~F} 5, \mathrm{G} 1, \mathrm{G} 2, \mathrm{G} 3, \mathrm{H} 1, \mathrm{H} 2 \\
\text { 2A, 2J1, 2J2, 2N, 2Q, 2S, 2U, 2V, } \\
\text { 2Z, 3M, 3V, 4B, 4G1, 4G2, TC46 }\end{array}$ & Heterozygous & - & c.3470T $>C$ & p.I1157T & - & - \\
\hline & $3 Z$ & Heterozygous & - & c. $3478 \mathrm{G}>\mathrm{A}$ & p.E1160K & - & - \\
\hline \multirow[t]{9}{*}{$\mathrm{CFH}$} & $\mathrm{TC} 20^{\mathrm{a}}$ & Heterozygous & rs762132970 & c. $526 \mathrm{~T}>\mathrm{C}$ & p.F176L & - & - \\
\hline & TC36 & Heterozygous & - & c. $695 \mathrm{G}>\mathrm{A}$ & p.R232Q & - & - \\
\hline & $\mathrm{TC}_{1} 4^{\mathrm{a}}$ & Heterozygous & - & c. $1951 \mathrm{C}>\mathrm{T}$ & p.H651Y & - & - \\
\hline & TC7 & Heterozygous & rs55931547 & c. $2392 \mathrm{G}>\mathrm{A}$ & p.D798N & 0.002 & - \\
\hline & TC33 & Heterozygous & - & c. $3572 \mathrm{C}>\mathrm{G}$ & p.S1191W & - & - \\
\hline & TC14 & Heterozygous & - & c. $3593 \mathrm{~A}>\mathrm{T}$ & p.E1198V & - & - \\
\hline & $\mathrm{TC} 1$ & Heterozygous & - & c. $3594 \mathrm{~A}>\mathrm{T}$ & p.E1198D & - & - \\
\hline & 2M, TC8 & Heterozygous & rs121913051 & c. $3643 \mathrm{C}>\mathrm{G}$ & p.R1215G & - & - \\
\hline & X, 2I, TC20 & Heterozygous & - & c. $3644 \mathrm{G}>\mathrm{A}$ & p.R1215Q & - & - \\
\hline THBD & $2 \mathrm{I}^{\mathrm{a}}$ & Heterozygous & - & c. $1499 \mathrm{C}>\mathrm{T}$ & T500M & - & - \\
\hline \multirow[t]{6}{*}{ MCP } & M1, M2 & Homozygous & - & c. $191 \mathrm{G}>\mathrm{A}$ & p.C64Y & - & - \\
\hline & $\mathrm{F}^{\mathrm{a}}, \mathrm{F}^{\mathrm{a}}, \mathrm{TC} 43$ & Heterozygous & rs116800126 & c. $293 \mathrm{C}>\mathrm{T}$ & p.T98I & 0.0036 & 0.001 \\
\hline & $\mathrm{P}$ & Homozygous & - & c.509delA & p.N170Mfs ${ }^{\mathrm{a}} 9$ & - & - \\
\hline & $2 \mathrm{D}$ & Heterozygous & rs202071781 & c. $565 \mathrm{~T}>\mathrm{G}$ & p.Y189D & 0.000008 & 0.0002 \\
\hline & $2 \mathrm{U}^{\mathrm{a}}$ & Heterozygous & rs773860894 & c. $.583 \mathrm{C}>\mathrm{T}$ & p.P195S & 0.00002 & - \\
\hline & $2 \mathrm{D}^{\mathrm{a}}$ & Heterozygous & rs767322836 & c. $1076 \mathrm{C}>\mathrm{T}$ & p.A359V & 0.00002 & - \\
\hline \multirow[t]{2}{*}{ DGKE } & $2 \mathrm{R}$ & Heterozygous & - & c.71delT & p.L24Cfs ${ }^{\mathrm{a}} 145$ & - & - \\
\hline & $2 \mathrm{R}$ & Heterozygous & - & c. $1213-2 \mathrm{~A}>\mathrm{G}$ & r.spl? p.(A405_E428del) & - & - \\
\hline
\end{tabular}

$M A F$ minor allele frequency

${ }^{\text {a } P a t i e n t s ~ w h o ~ h a d ~ a n o t h e r ~ c a n d i d a t e ~ a H U S-p r e d i s p o s i n g ~ v a r i a n t ~ w i t h ~ k n o w n ~ p a t h o g e n i c i t y ~}$

frequencies of $\mathrm{CFH}$ variants in Japanese patients compared with Caucasians $[2,9,16]$. These findings agree with those of cohorts from East Asia with limited age distributions or small sample sizes [17, 18]. Moreover, in this study, we expanded our aHUS previous cohort [4], and reconfirmed that $C 3$ variants are frequent in Japan, and demonstrated that the most common genetic abnormality was $C 3$ p.I1157T. This variant has not been found among East Asian and Japanese healthy populations. Thus, we revealed that genetic backgrounds differ by region and race.

Patients with the unique $C 3$ p.I1157T variant were notable not only for their high frequency of relapses, but also for their favorable prognosis; $92 \%$ of them remained in remission (median and average follow-up was 21 and 22 years, respectively) despite repeated relapse episodes. Relapses could usually be treated successfully with just supportive care, and in that respect differed from relapses of patients with other $C 3$ variants. These findings not only reaffirm the importance of characterizing the genotype-phenotype correlation in aHUS $[2,9,19,20]$, but also suggest that clinical presentation can be influenced differently by particular variants in the same defective protein. Furthermore, this finding raises a recently debated issue [21], namely, the permanent use of eculizumab for managing aHUS. Our study of $C 3$ p.I1157T indicates the importance of understanding differences in the natural history of aHUS and treatment duration and decision of discontinuation might depend on individual genetic variations.

Since there is no objective test for diagnosing aHUS except for time-consuming genetic analysis, diagnosis 
Table 3 Treatment and outcome of patients with aHUS according to each complement abnormality

\begin{tabular}{|c|c|c|c|c|c|c|c|c|}
\hline \multirow[t]{2}{*}{ Patients } & \multirow[t]{2}{*}{ Overall } & \multicolumn{4}{|c|}{ Genetic abnormalities } & \multirow[t]{2}{*}{ Anti-CFH Abs } & \multirow[t]{2}{*}{ Unidentified } & \multirow[t]{2}{*}{ Unanalyzed } \\
\hline & & $C 3$ & $\mathrm{CFH}$ & $M C P$ & $D G K E$ & & & \\
\hline Patients $(n)$ & 118 & 32 & 10 & 5 & 1 & 20 & 36 & 14 \\
\hline Follow-up ${ }^{a}$ (years) & $2.3(0-54.0)$ & $17.0(0.1-54.0)$ & $1.6(0.5-9.0)$ & $11.0(0.3-29.0)$ & 1.5 & $2.0(0-25.0)$ & $1.0(0-6.0)$ & $1.5(0-37.0)$ \\
\hline \multicolumn{9}{|l|}{ Treatment $(n)$} \\
\hline $\begin{array}{l}\text { At initial onset: } \mathrm{Ob} / \\
\mathrm{PT} / \mathrm{ECZ}_{ \pm} \mathrm{PT}^{\mathrm{b}}\end{array}$ & $26 / 41 / 34$ & $12 / 10 / 3$ & $1 / 3 / 6$ & $1 / 3 / 0$ & $0 / 0 / 1$ & $0 / 10 / 9$ & $9 / 10 / 15$ & $3 / 5 / 0$ \\
\hline $\begin{array}{l}\text { Before approval of } \\
\text { ECZ }\end{array}$ & $20 / 23 / 6$ & $11 / 7 / 0$ & $1 / 2 / 0$ & $1 / 2 / 0$ & $0 / 0 / 1$ & $0 / 6 / 2$ & $4 / 3 / 3$ & $3 / 3 / 0$ \\
\hline $\begin{array}{l}\text { After approval of } \\
\text { ECZ }\end{array}$ & $6 / 18 / 28$ & $1 / 3 / 3$ & $0 / 1 / 6$ & $0 / 1 / 0$ & - & $0 / 4 / 7$ & $5 / 7 / 12$ & $0 / 2 / 0$ \\
\hline $\begin{array}{l}\text { Current: non-use/use } \\
\text { of ECZ }\end{array}$ & $81 / 27$ & $26 / 6$ & $3 / 7$ & $5 / 0$ & $0 / 1$ & $14^{\mathrm{d}} / 4$ & $22 / 9$ & $11 / 0$ \\
\hline \multicolumn{9}{|c|}{ Outcome at discharge after initial onset, $n(\%)$} \\
\hline Remission & $95(84)$ & $30(94)$ & $6(60)$ & $5(100)$ & $1(100)$ & $17(89)$ & $25(71)$ & $11(100)$ \\
\hline ESRD & $14(12)$ & $1(3)$ & $3(30)$ & $0(0)$ & $0(0)$ & $2(11)$ & $8(23)$ & $0(0)$ \\
\hline Death & $4(4)$ & $1(3)$ & $1(10)$ & $0(0)$ & $0(0)$ & $0(0)$ & $2(6)$ & $0(0)$ \\
\hline \multicolumn{9}{|l|}{ Long-term outcome ${ }^{a}$} \\
\hline Relapse (\%) & 38 & 77 & 33 & 50 & 100 & 0 & 4 & 73 \\
\hline Number of relapses & $0(0-9)$ & $2(0-9)$ & $0(0-2)$ & $0(0-4)$ & 1 & 0 & $0(0-1)$ & $2(0-5)$ \\
\hline $\begin{array}{l}\text { Relapse within a } \\
\text { year of initial } \\
\text { onset }(\%)\end{array}$ & 36 & 23 & 100 & 0 & - & - & 0 & 60 \\
\hline $\begin{array}{l}\text { Time until the sec- } \\
\text { ond onset }^{c} \text { (years) }\end{array}$ & $3.0(0.3-13.0)$ & $3.0(0.4-13.0)$ & 0.3 & $7.0(5.0-9.0)$ & NA & - & NA & $1.0(0.5-13.0)$ \\
\hline $\begin{array}{l}\text { Treatment for } \\
\text { relapses: } \mathrm{Ob} / \mathrm{PT} / \\
\mathrm{ECZ}_{ \pm} \mathrm{PT}^{\mathrm{b}}\end{array}$ & $6 / 12 / 8$ & $5 / 8 / 5$ & $0 / 1 / 2$ & $0 / 2 / 0$ & $0 / 0 / 1$ & - & $0 / 1 / 0$ & $1 / 2 / 0$ \\
\hline \multicolumn{9}{|c|}{ Outcome reported on latest questionnaire, n (\%) } \\
\hline Remission & $94(83)$ & $26(81)$ & $7(70)$ & $5(100)$ & $1(100)$ & $19(100)$ & $25(71)$ & $11(100)$ \\
\hline ESRD & $13(12)$ & $5(16)$ & $2(20)$ & $0(0)$ & $0(0)$ & $0(0)$ & $6(17)$ & $0(0)$ \\
\hline Death & $6(5)$ & $1(3)$ & $1(10)$ & $0(0)$ & $0(0)$ & $0(0)$ & $4(11)$ & $0(0)$ \\
\hline
\end{tabular}

Results are expressed as numerical values, median (range) for continuous variables and percentages for categorical variables unless otherwise indicated

$O b$ observational therapy, $P T$ plasma therapy (plasma infusion or plasma exchange), ECZ eculizumab, ESRD end-stage renal disease (requiring renal replacement therapy), $N A$ not available

${ }^{a}$ Long-term outcome was based on the latest questionnaire

${ }^{b}$ Patients categorized in the ECZ group were treated with eculizumab with or without plasma therapy

${ }^{\mathrm{c}}$ Relapses after transplantation are not included

${ }^{\mathrm{d}}$ Eight out of 13 patients were receiving immunosuppressive therapy

depends on recording clinical manifestations and basic laboratory data at the first visit, especially for initial disease onset. The following three findings provide additional clues supporting its clinical diagnosis. First, nearly, half of aHUS patients, regardless of their underlying pathogenic abnormalities, presented with severe thrombocytopenia $\left(<3 \times 10^{4}\right.$ / $\mu \mathrm{l})$. While this condition is a well-known characteristic of TTP in thrombotic microangiopathy [22], our data suggest that it may not only indicate TTP, but also aHUS. Second, many patients exhibited severe hematuria and proteinuria. Although those with $D G K E$ variants are known to manifest nephrotic syndrome [12], we observed severe proteinuria in up to $50 \%$ of patients with other genetic abnormalities. Finally, aHUS patients showed activation of the coagulation and fibrinolytic systems without severe consumption of clotting factors. Even though differentiating between DIC and aHUS has been controversial [23], our laboratory data indicated that most aHUS patients do not meet the criteria for DIC.

Furthermore, we reported findings regarding practical treatments and their outcomes not only in the acute phase, but also over relatively long periods. Renal outcomes in 
Fig. 2 Cumulative renal survival of aHUS patients according to each abnormality estimated by Kaplan-Meier analyses. a Patients with $M C P$ variants and anti-CFH antibodies had never reached end-stage renal disease. Patients with $C 3$ variants had good renal outcomes, in contrast with patients with $\mathrm{CFH}$ variants and patients in the unidentified group. b Among patients with $C 3$ variants, patients with the C3 p.I1157T variant had better renal survival than patients with $p=0.0003)$. Anti-CFH Abs anti$\mathrm{CFH}$ antibodies other $C 3$ variants (log-rank test,
A

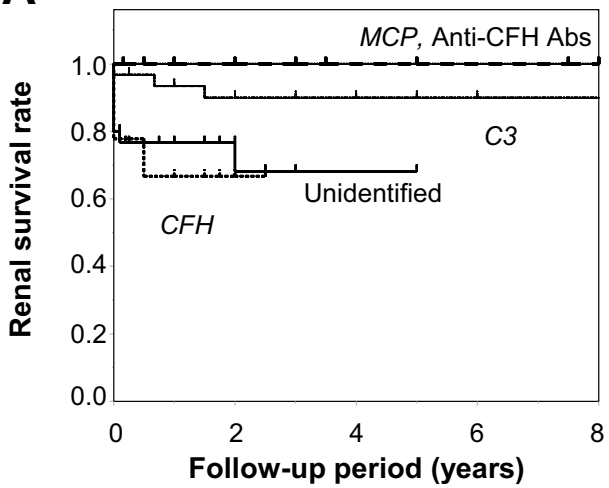

B

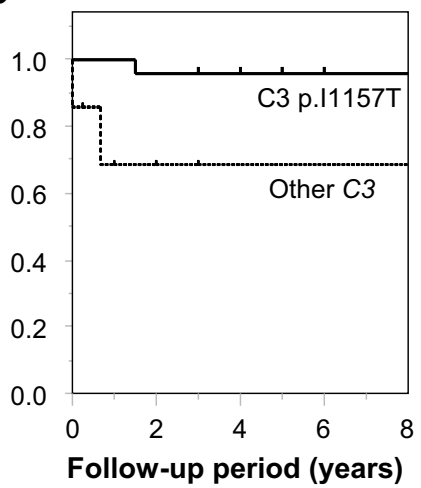

Number at risk

$\begin{array}{lc}\text { C3 } & 31 \\ \text { CFH } & 9 \\ \text { MCP } & 4 \\ \text { Anti-CFH Abs } & 19 \\ \text { Unidentified } & 30\end{array}$

26
1
3
11
9

Japanese patients were better than those in Caucasian patients [9, 24], even before eculizumab was introduced. This is probably due to the unique genetic background of the Japanese population, specifically the predominance of C3 p.I1157T and anti-CFH antibodies. This background also likely explains the lack of significant differences between the outcomes of patients treated with eculizumab (overall renal mortality rate: $20 \%$ ) and without eculizumab (renal mortality rates of patients treated with observational therapy and plasma therapy: 13 and $9 \%$, respectively) ( $p=0.22$, Online Resource 2). The reason we observed better outcomes in patients with anti-CFH antibodies compared to previous reports $[2,25]$ is unclear, but it too may be due to the unique characteristics of patients in Japan. The optimal duration of eculizumab use and possible withdrawal effects are ongoing questions in the management of patients with various aHUS abnormalities [21,26] and our report of 13 cases of eculizumab discontinuation could provide new insight into this question.

One limitation of this study is that aHUS was not a wellunderstood entity in clinics approximately 20 years ago, when we started enrolling aHUS patients in Japan (Online Resource 6) $[5,27]$. Thus, patients who eventually required permanent RRT might not have been enrolled in this study, which may have improved the prognosis in our cohort. Actually, the risk for ESRD and death did not change before and after the approval of eculizumab (13 and 19\%, respectively). Another limitation is that this was an observational study and the prognosis could have change with the development of treatment for aHUS.

In conclusion, our study describes the genetic and clinical features of Japanese patients with aHUS, and is the first analysis of this topic in a large cohort of Asian patients. Our results suggest that the etiologies of aHUS might differ among continents and races. The $C 3$ p.I1157T variant was predominant in Japan, was associated with a favorable prognosis, and differed from other previously reported $C 3$ variants. Our findings emphasize the importance of a detailed understanding of each patient's genotype and its corresponding clinical characteristics, as this knowledge is necessary to help determine treatment strategies, including eculizumab discontinuation. 
Table 4 Comparison of patients with $C 3$ p.I1157T and other $C 3$ variants

\begin{tabular}{|c|c|c|c|}
\hline Patients with $\mathrm{C} 3$ variants & p.I1157T & Other variants & $p$ value \\
\hline Patients $(n)$ & 24 & 8 & \\
\hline Age at initial onset (years) & $6.0(1.0-70.0)$ & $5.4(0.3-48.0)$ & 0.57 \\
\hline Children $(<18$ years of age) $(\%)$ & 67 & 63 & 0.99 \\
\hline Male (\%) & 71 & 50 & 0.40 \\
\hline Family history of aHUS (\%) & 58 & 13 & 0.041 \\
\hline Follow-up ${ }^{\text {a }}$ (years) & $21.0(3.0-54.0)$ & $2.3(0.1-35.0)$ & 0.012 \\
\hline \multicolumn{4}{|l|}{ Clinical presentation (\%) } \\
\hline \multicolumn{4}{|l|}{ Anemia } \\
\hline $\mathrm{Hb}<10 \mathrm{~g} / \mathrm{dl}$ & 29 & 100 & 0.021 \\
\hline $\mathrm{Hb}<6 \mathrm{~g} / \mathrm{dl}$ & 0 & 17 & 0.46 \\
\hline \multicolumn{4}{|l|}{ Thrombocytopenia } \\
\hline $\mathrm{Plt}<15 \times 10^{9} / \mu \mathrm{l}$ & 100 & 100 & \\
\hline $\mathrm{Plt}<3 \times 10^{9} / \mu \mathrm{l}$ & 57 & 33 & 0.59 \\
\hline AKI & 100 & 100 & \\
\hline AKI requiring RRT & 41 & 50 & 0.99 \\
\hline Reduced C3 & 0 & 100 & 0.008 \\
\hline \multicolumn{4}{|l|}{ Treatment } \\
\hline \multicolumn{4}{|l|}{ At initial onset } \\
\hline $\mathrm{Ob} / \mathrm{PT} / \mathrm{ECZ}^{\mathrm{b}}$ & $11 / 6 / 0$ & $1 / 4 / 3$ & 0.008 \\
\hline Before approval of $\mathrm{ECZ}^{\mathrm{b}}$ & $10 / 5 / 0$ & $1 / 2 / 0$ & \\
\hline After approval of $E C Z^{b}$ & $1 / 1 / 0$ & $0 / 2 / 3$ & \\
\hline \multicolumn{4}{|l|}{ Current } \\
\hline Non-use/use of ECZ & $21 / 3$ & $5 / 3$ & 0.12 \\
\hline Outcome at discharge after initial onset, $n(\%)$ & & & 0.041 \\
\hline Remission & $24(100)$ & $6(75)$ & \\
\hline ESRD & $0(0)$ & $1(13)$ & \\
\hline Death & $0(0)$ & $1(13)$ & \\
\hline \multicolumn{4}{|l|}{ Long-term outcome $^{\mathrm{a}}$} \\
\hline Relapse $(\%)$ & 88 & 33 & 0.016 \\
\hline Number of relapses & $2(0-9)$ & $0(0-3)$ & 0.003 \\
\hline Relapse within a year of initial onset (\%) & 25 & 0 & 0.99 \\
\hline Time until the second onset ${ }^{\mathrm{c}}$ (years) & $3.0(0.4-13.0)$ & 4.0 & 0.38 \\
\hline Treatment for relapses: Ob/PT/ECZ $\pm \mathrm{PT}^{\mathrm{b}}$ & $5 / 7 / 5$ & $0 / 1 / 0$ & 0.86 \\
\hline Outcome reported on latest questionnaire, $n(\%)$ & & & 0.023 \\
\hline Remission & $22(92)$ & $4(50)$ & 0.041 \\
\hline ESRD & $2(8)$ & $3(38)$ & 0.012 \\
\hline Death & $0(0)$ & $1(13)$ & \\
\hline
\end{tabular}

Results are expressed as numerical values, median (range) for continuous variables and percentages for categorical variables unless otherwise indicated

HUS hemolytic uremic syndrome, $H b$ hemoglobin, Plt platelets, AKI acute kidney injury, RRT renal replacement therapy, $O b$ observational therapy, $P T$ plasma therapy (plasma infusion or plasma exchange), $E C Z$ eculizumab, ESRD end-stage renal disease (requiring renal replacement therapy)

${ }^{a}$ Long-term outcome was based on the latest questionnaire

${ }^{b}$ Patients categorized in the ECZ group were treated with eculizumab with or without plasma therapy

${ }^{c}$ Relapses after transplantation are not included
Acknowledgements We thank all the participants in this registry and the clinicians who referred their patients and contributed to the sample collection and clinical data acquisition. We thank the following institutions for consulting more than two patients: Aichi Children's Health and Medical Center, Osaka Medical College, Osaka City General Hospital, Osaka Rosai Hospital, Japanese Red Cross Otsu 
Hospital, Kawasaki Municipal Hospital, Kyusyu University Hospital, Kyoto University Hospital, Japanese Red Cross Kyoto Daini Hospital, Kurobe City Hospital, Teine Keijinkai Hospital, National Center for Child Health and Development, Chiba Children's Hospital, Chukyo Hospital, Tenri Hospital, Tokyo Women's Medical University, Tokyo Metropolitan Children's Medical Center, Japanese Red Cross Nagoya Daini Hospital, Nara Medical University, and Japanese Red Cross Fukuoka Hospital.

Author contributions $\mathrm{HK}$ and MN conceived and designed the clinical study; YY helped design the study and collect data. TU helped design and performed statistical analyses. M.T. helped formulation of registration system of UHCT ACReSS (University Hospital Clinical Trial Alliance in Japan). MF and HW helped collection of clinical data of some patients. YU, KK, and TM performed genetic analyses. $\mathrm{MM}$ and YF helped design the study and collect data. All authors were involved in writing the paper and had final approval of the submitted and published versions.

Funding This study was supported by research grants from the Ministry of Health, Labour, and Welfare of Japan (MN, HK, and TM); the Japan Society for the Promotion of Science, Grant-in-Aid for Scientific Research (C) (15K09246, HK, 16K09834, TM); the Japanese Association for Complement Research (HK); the Takeda Scientific Foundation (TM), and the Practical Research Project for Rare/Intractable Diseases from Japan Agency for Medical Research and Development, AMED (17ek0109254h0001, HK, MN, TM, MM and YY).

\section{Compliance with ethical standards}

Conflict of interest Yoshihiro Fujimura is a recipient of patent royalty for ADAMTS13 activity-ELISA kits from Alfressa, Honoraria: Hideki Kato and Masaomi Nangaku from Alexion Pharma, Hideo Wada from IL Japan and Alexion Pharma, and Masanori Matsumoto from Asahi Kasei Pharma and Alexion Pharma, Research funding: Masanori Matsumoto from Bayer Yakuhin, Chugai Pharmaceutical, and Asahi Kasei Pharma, Subsidies or Donations: Masaomi Nangaku from Alexion Pharma.

Research involving human participants and/or animals Ethical approval: all procedures performed in studies involving human participants were in accordance with the ethical standards of the institutional and/or national research committee at which the studies were conducted (IRB approval number G10029) and with the 1964 Helsinki declaration and its later amendments or comparable ethical standards.

Informed consent Informed consent was obtained from all individual participants included in the study.

Open Access This article is distributed under the terms of the Creative Commons Attribution 4.0 International License (http://creativecommons.org/licenses/by/4.0/), which permits use, duplication, adaptation, distribution and reproduction in any medium or format, as long as you give appropriate credit to the original author(s) and the source, provide a link to the Creative Commons license and indicate if changes were made.

\section{References}

1. Noris M, Remuzzi G. Atypical hemolytic-uremic syndrome. N Engl J Med. 2009;361(17):1676-87.
2. Noris M, Caprioli J, Bresin E, Mossali C, Pianetti G, Gamba S, et al. Relative role of genetic complement abnormalities in sporadic and familial aHUS and their impact on clinical phenotype. Clin J Am Soc Nephrol. 2010;5(10):1844-59.

3. Matsumoto T, Fan X, Ishikawa E, Ito M, Amano K, Toyoda H, et al. Analysis of patients with atypical hemolytic uremic syndrome treated at the Mie University Hospital: concentration of C3 p.I1157T mutation. Int J Hematol. 2014;100(5):437-42.

4. Yoshida Y, Miyata T, Matsumoto M, Shirotani-Ikejima H, Uchida $\mathrm{Y}$, Ohyama Y, et al. A novel quantitative hemolytic assay coupled with restriction fragment length polymorphisms analysis enabled early diagnosis of atypical hemolytic uremic syndrome and identified unique predisposing mutations in Japan. PLoS One. 2015;10(5): e0124655.

5. Kato H, Nangaku M, Hataya H, Sawai T, Ashida A, Fujimaru R, et al. Clinical guides for atypical hemolytic uremic syndrome in Japan. Clin Exp Nephrol. 2016;20(4):536-43.

6. Fan X, Yoshida Y, Honda S, Matsumoto M, Sawada Y, Hattori $\mathrm{M}$, et al. Analysis of genetic and predisposing factors in Japanese patients with atypical hemolytic uremic syndrome. Mol Immunol. 2013;54(2):238-46.

7. Miyata T, Uchida Y, Ohta T, Urayama K, Yoshida Y, Fujimura Y. Atypical haemolytic uraemic syndrome in a Japanese patient with DGKE genetic mutations. Thromb Haemost. 2015;4:862-3

8. Kobayashi N, Maekawa T, Takada M, Tanaka H, Gonmori H. Criteria for diagnosis of DIC based on the analysis of clinical and laboratory findings in 345 DIC patients collected by the Research Committee on DIC in Japan. Bibl Haematol. 1983;49:265-75.

9. Fremeaux-Bacchi V, Fakhouri F, Garnier A, Bienaimé F, DragonDurey MA, Ngo S, et al. Genetics and outcome of atypical hemolytic uremic syndrome: a nationwide French series comparing children and adults. Clin J Am Soc Nephrol. 2013;8(4):554-62.

10. Manuelian T, Hellwage J, Meri S, Caprioli J, Noris M, Heinen $\mathrm{S}$, et al. Mutations in factor $\mathrm{H}$ reduce binding affinity to $\mathrm{C} 3 \mathrm{~b}$ and heparin and surface attachment to endothelial cells in hemolytic uremic syndrome. J Clin Investig. 2003;111(8):1181-90.

11. Fremeaux-Bacchi V, Moulton EA, Kavanagh D, Dragon-Durey MA, Blouin J, Caudy A, et al. Genetic and functional analyses of membrane cofactor protein (CD46) mutations in atypical hemolytic uremic syndrome. J Am Soc Nephrol. 2006;17(7):2017-25.

12. Lemaire M, Frémeaux-Bacchi V, Schaefer F, Choi M, Tang WH, Le Quintrec M, et al. Recessive mutations in DGKE cause atypical hemolytic-uremic syndrome. Nat Genet. 2013;45(5):531-6.

13. Sánchez Chinchilla D, Pinto S, Hoppe B, Adragna M, Lopez L, Justa Roldan ML, et al. Complement mutations in diacylglycerol kinase- $\varepsilon$-associated atypical hemolytic uremic syndrome. Clin J Am Soc Nephrol. 2014;9(9):1611-9.

14. Ohta T, Urayama K, Tada Y, Furue T, Imai S, Matsubara K, et al. Eculizumab in the treatment of atypical hemolytic uremic syndrome in an infant leads to cessation of peritoneal dialysis and improvement of severe hypertension. Pediatr Nephrol. 2015;30(4):603-8.

15. Toyoda H, Wada H, Miyata T, Amano K, Kihira K, Iwamoto $\mathrm{S}$, et al. Disease recurrence after early discontinuation of eculizumab in a patient with atypical hemolytic uremic syndrome with complement C3 I1157T mutation. J Pediatr Hematol Oncol. 2016;38(3):e137-9.

16. Maga TK, Nishimura CJ, Weaver AE, Frees KL, Smith RJ. Mutations in alternative pathway complement proteins in American patients with atypical hemolytic uremic syndrome. Hum Mutat. 2010;31(6):E1445-60.

17. Lee JM, Park YS, Lee JH, Park SJ, Shin JI, Park YH, et al. Atypical hemolytic uremic syndrome: Korean pediatric series. Pediatr Int. 2015;57(3):431-8. 
18. Zhang T, Lu J, Liang S, Chen D, Zhang H, Zeng C, et al. Comprehensive analysis of complement genes in patients with atypical hemolytic uremic syndrome. Am J Nephrol. 2016;43(3):160-9.

19. Malina M, Roumenina LT, Seeman T, Le Quintrec M, DragonDurey MA, Schaefer F, et al. Genetics of hemolytic uremic syndromes. Presse Med. 2012;41(3 Pt 2):e105-14.

20. Sellier-Leclerc AL, Fremeaux-Bacchi V, Dragon-Durey MA, Macher MA, Niaudet P, Guest G, et al. Differential impact of complement mutations on clinical characteristics in atypical hemolytic uremic syndrome. J Am Soc Nephrol. 2007;18(8):2392-400.

21. Fakhouri F, Fila M, Provot F, Delmas Y, Barbet C, Chatelet V, et al. Pathogenic variants in complement genes and risk of atypical hemolytic uremic syndrome relapse after eculizumab discontinuation. Clin J Am Soc Nephrol. 2017;12(1):50-9.

22. Coppo P, Schwarzinger M, Buffet M, Wynckel A, Clabault K, Presne C, et al. Predictive features of severe acquired ADAMTS13 deficiency in idiopathic thrombotic microangiopathies: the French TMA reference center experience. PLoS One. 2010;5(4):e10208.

23. Kurosawa S, Stearns-Kurosawa DJ. Complement, thrombotic microangiopathy and disseminated intravascular coagulation. J Intensive Care. 2014;2(1):65.
24. Moake JL. Thrombotic microangiopathies. N Engl J Med. 2002;347(8):589-600.

25. Sinha A, Gulati A, Saini S, Blanc C, Gupta A, Gurjar BS, et al. Prompt plasma exchanges and immunosuppressive treatment improves the outcomes of anti-factor $\mathrm{H}$ autoantibodyassociated hemolytic uremic syndrome in children. Kidney Int. 2014;85(5):1151-60.

26. Goodship TH, Cook HT, Fakhouri F, Fervenza FC, FremeauxBacchi V, Kavanagh D, et al. Atypical hemolytic uremic syndrome and C3 glomerulopathy: conclusions from a "Kidney Disease: Improving Global Outcomes" (KDIGO) Controversies Conference. Kidney Int. 2017;91(3):539-51.

27. Sawai T, Nangaku M, Ashida A, Fujimaru R, Hataya H, Hidaka $Y$, et al. Diagnostic criteria for atypical hemolytic uremic syndrome proposed by the Joint Committee of the Japanese Society of Nephrology and the Japan Pediatric Society. Clin Exp Nephrol. 2014;18(1):4-9. 